\title{
Histoire et dialectique des idéologies et significations religieuses ${ }^{1}$
}

\author{
$\sim$ Albert Doja $\sim$
}

\begin{abstract}
The aim of this article is to consider the possibility of a new analytical methodology that could include multidisciplinary approaches into the study of religious ideologies and practices, taking benefit from historical and ethnographic interpretations, but also from linguistic and philological, psychoanalytical, philosophical, sociological, and anthropological considerations. If one tries to study the role or function played by religion in society, considering human beings as builders of symbolic worlds, one should set out to analyze, if not rules, at least general processes of the religious readjustment with respect to the rest of social life, whenever the gap is much too wide, or the religious transfer from one realm to another entirely different.
\end{abstract}

\section{INTRODUCTION}

L'objet de cet article porte essentiellement sur l'éventualité de nouvelles démarches qui puissent intégrer dans l'étude des idéologies et pratiques religieuses des approches pluridisciplinaires, tirant profit non seulement des interprétations historiques et ethnographiques, mais aussi des considérations sociolinguistiques et philologiques, psychanalytiques, philosophiques, sociologiques, anthropologiques, etc. Si l'on veut étudier le rôle ou la fonction que la religion joue dans la société, tout en considérant l'homme en tant que constructeur de mondes symboliques, il faut s'attacher à chercher, sinon des lois, du moins des processus généraux, comme le rééquilibrage du religieux par rapport au reste de la vie sociale, chaque fois que l'écart est trop grand, ou le transfert religieux d'un domaine à un autre entièrement différent. Quoique le détour puisse parâtre un peu long, pour essayer de donner, non une réponse générale, mais la réponse qui convient à un cas particulier envisagé, il faudrait en effet considérer plus profondément certaines caractéristiques de la religion qui de ce point de vue paraissent plus essentielles que d'autres.

\section{Scrupule Subjectif ou Lien Objectif}

L'examen d'un certain nombre de définitions courantes de la religion montre que pour atteindre à la compréhension des phénomènes religieux, il convient d'écarter quelques idées reçues, propres aux conceptions chrétiennes. Aujourd'hui, on a tendance à projeter l'idéologie chrétienne sur les autres religions en considérant toute croyance 
comme une foi ou une religion, au sens que ces mots ont pris chez les chrétiens. C'est une erreur qui empêche de comprendre les religions, leur signification et leur histoire. L'anthropologie religieuse a su opérer les décentrements propres à la prémunir contre ces erreurs de jugement. Les observations ethnographiques d'Evans-Pritchard, par exemple, selon lesquelles un certain nombre de langues naturelles, aussi riches soientelles en termes désignant des attitudes mentales, ne disposent pas de l'équivalent du concept occidental de religion ou de croyance, censé s'appliquer à une expérience ou à une aptitude universelle, ont une portée anthropologique générale.

Si une langue dispose ou non d'un terme explicite pour exprimer les réalités institutionnelles, c'est forcément un test de conceptualisation. Il est évident cependant que les items lexicaux ne sont pas toujours nécessaires en tant que tests de conceptualisation. Plusieurs langues disposent de catégories grammaticales qui signalent l'engagement des interlocuteurs vis-à-vis de l'exactitude d'une proposition communiquée, ainsi que différentes attitudes propositionnelles d'intentionnalité, qui confirment ou infirment la validité de la proposition et l'actualisation de la situation représentée. Néanmoins, dans le cas de la religion, c'est la nature même de cette notion de ne pas se prêter à une appellation unique et constante, puisque cette réalité omniprésente n'est pas toujours conçue comme une institution séparée. L'institution de la religion n'est pas nettement séparée des autres éléments, ni posée en dehors de l'ensemble institutionnel. Les civilisations anciennes, de même que les sociétés étudiées par les anthropologues, n'ont pas une religion. C'est leur constitution du social qui est intrinsèquement religieuse. L'ensemble du culturel est à peu près coextensif à l'ensemble du religieux, tout est religieux, tout est imbu de religion, tout est signe des forces divines. La société est tellement pénétrée de surnaturel qu'il n'est pas question d'isoler la sacralité de la socialité elle-même. ${ }^{2}$

Dans ces conditions, il n'est pas fortuit que les sociétés indo-européennes non plus n'avaient pas de terme pour désigner la religion. Encore à date historique, plusieurs langues indo-européennes en sont dépourvues. Le besoin de concevoir clairement et donc de recourir à un terme spécial pour dénommer la religion ne se fait sentir que lorsqu'elle est délimitée dans un domaine distinct, à partir du moment où l'on peut savoir ce qui lui appartient et ce qui lui est étranger. Une telle désignation apparaît, à travers des termes qui peuvent passer pour des équivalents de "religion," premièrement en grec et en latin. Les langues occidentales (à la différence d'autres idiomes, même indo-européens) ont donc appris, quoique assez tardivement, à spécialiser un vocable pour distinguer l'appareil des croyances et des rites des autres institutions sociales. Or c'est là une initiative de rupture, qui tend à penser séparément ce qui ne l'avait jamais été.

À l'origine, en latin, religio ne désigne pas la "religion" dans son ensemble, mais l'astreinte aux obligations du culte. Terme de la langue augurale, il désigne un "scrupule relatif aux omina," à ces déclarations de vérité, présages vrais, signes du destin, paroles de bon augure, annonciatrices du sort. Le terme désigne donc une disposition subjective. Tel apparaît aussi le trait dominant de religio dans ses exemples "laïcisés." De là vient également l'expression religio est, "avoir scrupule." À l'époque classique, les emplois où religio reprend scrupulus sont en effet très fréquents, souvent en relation avec des phénomènes religieux aussi. Pour Cicéron, les religiosi sont ceux qui reprennent diligemment toutes les choses qui se rapportent au culte des dieux (De Natura Deorum II, 28: 72). Le sens de religio, qu'on retrouverait dans quantité d'exemples, c'est 
accomplir un culte "avec le plus méticuleux souci des rites," alors que le dérivé religiosus signifie "scrupuleux à l'égard du culte, se faisant un cas de conscience des rites."

Au total, d'après Émile Benveniste (1969, 2: 265-72), religio serait plutôt une hésitation qui retient, un scrupule qui empêche, et non un sentiment qui dirige vers une action ou qui incite à pratiquer le culte. De même, les données se rapportant au terme grec threskeía s'enchaînent et complètent une notion comparable. Il se rattache à un thème verbal marquant l'attention au rite, la préoccupation d'être fidèle à une règle. Le sens du latin religio, démontré par l'usage ancien sans la moindre ambiguïté, impose donc une seule interprétation, celle signalée par Cicéron et appuyée de son autorité, religio dérive de legere, "cueillir, ramasser" ou de religere, "recueillir, recollecter." Quoique ce dernier verbe soit attesté par un seul participe, au moyen de divers restitutions, d'après Benveniste, il voulait dire revenir sur ce qu'on fait, reprendre pour un nouveau choix, ressaisir par la pensée ou la réflexion, redoubler d'attention et d'application. D'après Dumézil $(1987,145)$, il paraît que le concept opposé à religio se trouve effectivement dans le verbe negligere aussi.

En conséquence, religio est bien synonyme de scrupule, de soin méticuleux, de ferveur inquiète. Dans ce sens, le mot convient éminemment à l'exercice du culte, à l'observance rituelle, exigeant que la pratique soit littérale, le zèle soucieux et vigilant. Recommencer un choix déjà fait, réviser la décision qui en résulte, tel est le sens propre de religio. Il indique une disposition intérieure et non une propriété objective de certaines choses ou un ensemble de croyance et de pratiques. La religio romaine à l'origine est essentiellement subjective (Benveniste 1969, 2: 271-2). Si religio équivaut à délicatesse de conscience, à recueillement intense, à circonspection craintive et minutieuse, on comprend que le terme se soit fixé rapidement, presque exclusivement, sur l'expérience ou la manipulation du sacré. Il ne la signifiait pas d'abord. En signifiant l'attitude qu'elle requiert, il était destiné à glisser de la disposition subjective qu'il souligne aux réalités objectives auxquelles cette disposition se réfère.

Il n'est pas fortuit qu'à la suite de Tertullien, rhéteur latin converti au christianisme assez tôt, au tournant des $2^{\mathrm{e}} 3^{\mathrm{e}}$ siècles, et considéré comme le premier Père de l'Église d'Occident, les auteurs chrétiens se plaisent à expliquer le latin religio par les verbes ligare, religare "lier, relier." Il ne s'agit pas ici d'une simple étymologie populaire, d'autant plus que, morphologiquement, il est beaucoup plus difficile de ramener le mot à ligare. Lactance, un autre rhéteur latin converti au christianisme au début du $4^{\mathrm{e}}$ siècle, insiste expressément: "le terme de religio a été tiré du lien de la piété, parce que Dieu se lie l'homme et l'attache par la piété." On est donc en présence d'un changement du contenu de la religion, qui est d'autant plus intéressant qu'il témoigne d'une idée toute différente de la vieille religio romaine et qui prépare déjà l'acception moderne de la religion comme une idéologie préconstruite et imposée de l'extérieur. Le concept de religio est remodelé sur l'idée que l'homme se fait alors de sa relation à Dieu. Pour un chrétien, ce qui caractérise la nouvelle foi par rapport aux cultes païens, c'est le lien de la piété, cette dépendance du fidèle vis-à-vis de Dieu, cette obligation, qui est un lien objectif, au sens propre du mot. La religion signifierait alors "attache" ou "dépendance" et elle profiterait même des nuances et des variations de sens qu'on obtient, en français comme en latin, lorsqu'on change un rattachement en attachement, un lien effectif en lien affectif. Et cette opinion des Pères anciens de l'Église chrétienne est aussi adoptée par de nouveaux défenseurs (art. Religio, in: Pauly-Wissowa). 


\section{ReLigion et SUPERstition}

Il faudrait considérer pour acquis que toute religion se trouve à un moment ou un autre engagée dans un dialogue obligé avec d'autres traditions religieuses antérieures ou simultanées qu'elle essaie de combattre ou d'assimiler, ce qui produit le plus souvent des niveaux hiérarchiques entre conceptions spirituelles. Dans cette perspective, on peut essayer d'interroger en premier lieu les rapports que la religion chrétienne a entretenus avec les croyances et les pratiques dénoncées par l'Église comme "superstitieuses," mais qui ont constitué ce qu'en anthropologie sociale et religieuse on est convenu d'appeler "religion populaire." Ce qui va probablement nous permettre d'aborder ensuite le phénomène religieux en général, sa dynamique et son évolution au sein même d'une seule tradition religieuse, avant de conclure sur les rapports avec d'autres religions officiellement établies.

Le concept de religion populaire prend sa signification dans des sociétés où fonctionnent des autorités religieuses assurant une forte régulation de l'orthodoxie et de l'orthopraxie. La religion populaire est alors une religiosité vécue, en ce qui concerne représentations, affects et coutumes, sur le mode d'une différence par rapport à la religion officielle. Il s'agit d'un domaine très différencié, allant de pratiques violemment combattues par les autorités religieuses, telle la sorcellerie, jusqu'à des coutumes ou croyances largement intégrées dans les systèmes religieux officiels, tel le culte des saints guérisseurs, qui a tenu tant de place parmi les thérapeutiques populaires en pays de tradition catholique. À mesure que l'autorité orthodoxe condamne de telles traditions, celles-ci changent de caractère par rapport au système officiel, tout en continuant souvent avec leur forme et leur contenu antérieur. On devrait alors se demander dans quelle mesure ces traditions n'avaient pas déjà, au sein même de la religion officielle, une certaine autonomie et une signification différente de l'interprétation dogmatique qu'en donnaient les théologiens.

La superstition notamment est une notion curieuse et qui n'a pu naître que dans une civilisation et à une époque où l'esprit pouvait se détacher suffisamment des choses de la religion pour apprécier les formes normales et les formes exagérées de la croyance ou du culte. Cette notion indique que la pratique religieuse se complique d'observances de plus en plus minutieuses où interviennent la magie ainsi que des influences étrangères. La notion de "superstition" est fondamentale à la fois pour l'étude de la "religion populaire" et pour l'histoire de l'anthropologie des sociétés européennes en tant que discipline. C'est d'abord sous l'angle des superstitions qu'ont été collectées les observations sur la religion populaire.

Le terme de superstition dérive du latin superstitio, qui d'après sa forme devrait être un terme abstrait correspondant à superstes, "survivant." L'interprétation littérale conduirait donc à superstitio comme "survivance." Le terme indiquerait alors un "reste" d'une vieille croyance qui, à l'époque où on l'envisage, paraît superflue. Or cette explication, à laquelle ont aussi succombé certains folkloristes contemporains (Belmont 1979, 54-5), repose sur un contresens historique. Ce serait prêter aux anciens, et dès avant la tradition historique, l'attitude d'esprit et le sens critique du $19^{\mathrm{e}}$ siècle ou des ethnographes modernes, qui permettent de distinguer dans la religion des "survivances" d'une époque plus ancienne et qui ne s'harmonisent pas avec le reste. 
Selon Benveniste, le sens propre de superstes décrit le "témoin," soit comme celui "qui subsiste au-delà," témoin en même temps que survivant, soit comme "celui qui se tient sur la chose," qui y est présent. De là, superstitio, qualité de superstes, devait théoriquement signifier la "propriété d'être présent" en tant que "témoin." Mais superstitio est souvent associé à hariolatio "prédiction, prophétie, divination." Plus souvent encore c'est superstitiosus qui est constamment associé dans l'usage commun à hariolus, "devin." Toutefois c'est dans le vocabulaire spécial de la langue divinatoire que le terme a dû acquérir une signification technique de "présence" magique. La "vérité" du devin est le fait de "deviner" ce à quoi il n'a pas assisté. Alors superstitiosus est celui qui est "doué de la vertu de superstition," c'est-à-dire le devin, celui qui parle d'une chose passée comme s'il y avait réellement été. La "divination" ne s'applique pas ici au futur, mais au passé. La faculté de superstitio est le don de seconde vue qui permet de connaître le passé comme si on y avait été présent, superstes. Voilà pourquoi superstitiosus énonce la propriété de "double vue" qu'on attribue aux "voyants," celle d'être "témoin" d'événements auxquels on n'a pas assisté. Ainsi Benveniste a réussi à faire ordonner de façon régulière tous les termes: superstes, celui qui peut passer pour "témoin" pour avoir assisté à une chose accomplie; superstitio, "don de présence," faculté de témoigner comme si on y avait été; superstitiosus, celui qui est pourvu de ce “don de présence," qui lui permet d'avoir été dans le passé (1969, 2: 273-9).

Le sens moderne apparaît en dernier dans l'histoire sémantique du mot. De divination du passé, par extension de sens, superstitio est venu à désigner la divination en général et les diverses pratiques magiques qui lui sont apparentées. À un moment donné de l'évolution historique des mentalités, les pratiques divinatoires ont dû être tenues pour du charlatanisme. Les sorciers et les devins étaient méprisés, d'autant plus que pour la plupart, ils venaient à Rome de pays étrangers. Le don de superstitio, associé de ce fait à des pratiques réprouvées, a pris une connotation défavorable. Il a dénommé de bonne heure des pratiques d'une fausse religion considérées comme vaines et basses, indignes d'un esprit raisonnable. Les Romains, fidèles aux augures officiels, ont toujours condamné le recours à la magie, à la divination, à des pratiques jugées puériles. C'est alors, sur ce sens de “croyances religieuses méprisables," que s'est dégagée une nouvelle idée de superstitio, par antithèse avec religio, et qui a produit un nouveau sens pour superstitiosus, "superstitieux, qui s'adonne à la superstition, ou se laisse influencer par elle," entièrement distinct du premier sens et désormais antithétique de religiosus.

C'est déjà la vue éclairée, investie par le pouvoir institué des Romains rationalisants, qui a dissocié religio, le scrupule religieux, le culte authentique officiellement établi, de superstitio, en tant que forme dégradée et pervertie de la religion. Ainsi se précise déjà et dès cette époque le lien entre les deux valeurs successives de la notion de superstition, reflétant d'abord l'état des croyances populaires, puis l'attitude des Romains savants à l'égard de ces mêmes croyances par rapport à leur religion officielle. En tout cas, si la réflexion philosophique latine voyait dans la superstition une forme pervertie de la religion, elle n'accuse point un ou plusieurs cultes déterminés en dehors de la religion officielle. Pour Cicéron ou Sénèque, on trouve dans chaque peuple une religion et une superstition, ou, plus exactement, des esprits religieux et des esprits superstitieux, la religio étant, en dernière analyse, plus une attitude personnelle de l'individu qu'un contenu dogmatique. Prise en ce dernier sens, toutefois, elle est la part de vérité que renferme chaque religion particulière. 
De même, pour les autorités ecclésiastiques du christianisme, Lactance, Saint Augustin, Saint Thomas d'Aquin, J.-B. Thiers, la superstition est du côté de la religion, de la fausse religion sans doute, mais de la religion quand même. La superstition est perçue comme une forme exagérée, anormale, de la religion. Elle est une religion à quoi on ajoute quelque chose qui ne procède pas de Dieu, elle est un culte indu et superflu. Elle est une déviation de la religion, un ensemble de croyances et de rites procédant d'une attitude irrationnelle et traduisant une ignorance des lois de la nature et de la nature des dieux, alors que la véritable religion repose sur la connaissance de l'une et de l'autre. Ainsi, parce qu'elle est ignorance, la superstition est toujours "vaine" (vana), parce qu'elle est commandée par la crainte, elle est toujours "excessive" (nimia). Le superstitieux cherche à conjurer sa terreur par l'excès même des rites et du culte. Se constituant contre la raison, elle est une "fausse croyance," une "erreur pernicieuse," une "folie."

En revanche, en dehors des sphères philosophiques et ecclésiastiques, superstitio se charge d'une connotation fortement politique et ethnocentrique, désignant des cultes étrangers appréhendés comme globalement et foncièrement mauvais, parce que sentis comme une menace pour la société de l'époque. Le judaïsme et le christianisme, en particulier, seront qualifiés de superstitions. Religion étrangère ou religion d'une minorité, la superstition est bien devenue la religion des autres. Les chrétiens ont à leur tour taxé de superstitio les doctrines de leurs adversaires païens, mais aussi celles des juifs et des hérétiques.

Si par ailleurs c'est sous l'appellation de "superstition" que les faits folkloriques ont été repérés et recueillis, avant la naissance de l'ethnologie européenne, par les théologiens et les tenants du pouvoir de l'Église et ceux de la culture dite savante, ce n'a jamais été dans une intention anthropologique ou académique. Le terme même indique assez que le jugement de valeur porté par l'autorité religieuse dominante condamnait cette démarche. Plus tard, au cours du $19^{\mathrm{e}}$ siècle, s'est fait le passage à la notion de folklore religieux. Ainsi, en Angleterre, d'où le terme nous vient, on a regardé ces faits comme des "antiquités populaires," des erreurs et des opinions ridicules, caractéristiques du “commun" et du "vulgaire." Auparavant, en France, on les avait englobés sous le nom de "superstitions." À ces évaluations s'en ajouta ensuite une autre. On regarda les faits folkloriques comme des "témoins d'une époque archaïque" ou "ancienne," autrement dit comme des survivances (Van Gennep 1943). L'école anthropologique anglaise a ainsi mis à la disposition des savants la notion historico-scientifique de survivance et purifié, du même coup, l'atmosphère de toute préoccupation théologique (Saintyves 1932, 70). Cependant, si les notions de survivance et de superstition recouvraient plus ou moins les mêmes faits, cela ne veut pas dire que les termes aussi avaient le même sens, ou plutôt qu'ils désignaient la même attitude à l'égard des phénomènes observés. Tout au contraire, l'adoption de la notion de survivance dénote un changement d'attitude à l'égard des faits, qui n'est plus théologique ou ecclésiastique, mais se voulait plutôt scientifique et académique.

De même, avant Tylor et la vieille école d'anthropologie sociale britannique, les savants de l'Académie celtique en France, cherchant un substitut au terme de superstition, porteur d'une condamnation et d'un rejet, imaginent d'utiliser celui de "monument," qui fût positif et valorisant (Belmont 1986). Le monumentum est en latin notamment tout ce qui rappelle quelqu'un ou quelque chose, tout ce qui perpétue le 
souvenir, c'est la trace matérielle de ce qui est passé, de ce qui n'existe plus. C'est ensuite que le terme s'est figé dans le sens plus restreint du monument architectural commémoratif. Ce faisant, les savants de l'Académie celtique ont adopté une attitude propre à l'égard des faits, qui dans leur cas aussi se voulait être une attitude scientifique.

\section{Sens, Contrainte, Déplacement}

L'attitude théologique et ecclésiastique propre à la mentalité chrétienne à l'égard des croyances populaires n'est cependant pas dépourvue d'intérêt. Pour les auteurs chrétiens, il est essentiel de déterminer avec précision les règles qui permettent de reconnaître les superstitions. Dans son Traité des superstitions, l'abbé Jean-Baptiste Thiers postule qu'une chose est superstitieuse et illicite lorsqu'elle est accompagnée de certaines circonstances que l'on sait n'avoir aucune vertu naturelle pour produire les effets que l'on espère, par exemple la prononciation de certaines paroles inconnues (Lebrun 1976, 443-65). C'est une règle qu'il a reprise de Saint Thomas d'Aquin, qui s'exprime plus explicitement: Dans les choses qui se font pour produire quelques effets particuliers, il faut considérer si elles semblent pouvoir produire naturellement ces effets. Car si elles les peuvent produire, elles ne seront pas illicites, parce qu'il est permis de se servir de causes naturelles pour leur faire produire les effets qu'elles sont capables de produire. De sorte que si elles semblent ne pouvoir naturellement produire ces effets, il s'ensuit qu'on ne les emploie pas pour les produire comme des causes, mais seulement comme des signes. Et de cette manière, elles se rapportent aux pactes que l'on fait avec les démons. (Cité dans Belmont 1979, 58-9).

Les auteurs chrétiens tentent de prévenir l'objection que les croyances et les cérémonies de l'Église puissent être superstitieuses. Les cérémonies dont l'Église se sert, soit dans l'usage et l'administration des Sacrements, soit en d'autres circonstances, ne sont pas superstitieuses, bien qu'elles ne produisent pas naturellement les effets pour lesquels elles sont établies, pour la simple raison que l'Église a reçu de Dieu la puissance d'établir des cérémonies dont les effets ne peuvent donc venir que de Dieu. Si elles ne peuvent produire naturellement les effets qu'on en attend, c'est parce que, "par la vertu même qui leur a été instituée de Dieu," elles agissent comme des signes et non comme des causes naturelles.

La définition assez limitative de la superstition donnée par Thomas d'Aquin et les distinctions qu'il a opérées entre les formes licites et les formes illicites de certaines de ses variantes, telles que la divination ou les observances, devaient procurer un point d'appui à ce vaste effort par lequel la Renaissance allait justifier la plupart des superstitions. Ce mouvement, quoique puissant, n'était assurément ni général ni homogène. Les divers essais de légitimation étaient loin d'avoir le même sens et participaient de courants philosophiques parfois opposés, mais ils convergeaient dans leurs résultats. En tout cas, ces textes sont également remarquables en ce sens que les auteurs chrétiens placent les superstitions, et non sans raison, dans l'ordre du symbolique.

Les pratiques magiques et superstitieuses de la religion populaire ont l'air effectivement tout à fait dénuées de sens, donnant souvent une impression d'arbitraire et d'irrationalité. La spécificité psychologique du superstitieux se signale par une attitude cultuelle où il apparaît "tout occupé des cérémonies, sans y mettre de sainteté." Les 
anthropologues du $19^{\mathrm{e}}$ siècle pensaient même qu'on reconnaissait les superstitions précisément à leur absurdité. Cependant, si les croyances et les coutumes populaires, comme les cérémoniels individuels et obsessionnels, paraissent manquer de sens, c'est pour la raison simple qu'il s'agit dans leur cas d'un sens sitvé à un antre niveau ou d'un sens inconscient qu'il faudra redécouvrir.

En voyant dans toute religion une illusion, l'approche psychanalytique rend caduque une opposition de nature entre religion et superstition. Comme la religion, la superstition est une croyance dans la motivation de laquelle la réalisation d'un désir inconscient est prévalent. L'une et l'autre sont des constructions artificielles d'une réalité surnaturelle, fondées sur le mécanisme de projection, que la psychanalyse a pour fonction de retraduire en une psychologie de l'inconscient. ${ }^{3}$ La dimension anthropologique fondamentale de la religion, Freud la retrouve d'abord dans les rites religieux (1907), plus exactement dans l'analyse des rapports entre les pratiques religieuses et les actes compulsionnels des névrosés. Chez eux toute action, quelle qu'elle soit, peut devenir cérémonielle, si on la complique de petits ajouts et si on lui donne un rythme grâce à des pauses et à des répétitions (1976, 81-94). Analysant la parenté et la différence des rituels religieux avec les rites obsessionnels, Freud conclut que l'obsession est une caricature de la religion, alors que la religion ne serait qu'une névrose collective de l'humanité.

$\mathrm{Au}$ sens psychanalytique, la signification symbolique du rite est celle d'un acte déplacé, résultant d'un refoulement inconscient des pulsions primaires qu'opère la religion. Dans une telle psycho-histoire, la religion réalise spécifiquement le domaine symbolique où se fait le déplacement symptomatique social et culturel. Pour Freud, les actions compulsionnelles sont chargées de sens dans leurs moindres détails, elles sont au service d'intérêts importants de la personnalité et elles servent d'expression à des expériences vécues qui sont encore vivantes et à des pensées effectivement investies concernant des vécus. Dans ce cas comme dans celui des pratiques magiques et superstitieuses le sens est à interpréter soit historiquement, par le jeu fortuit des événements qui produisent la rencontre significative, soit symboliquement, c'est-à-dire agissant comme des signes.

À propos de la superstition obsessionnelle, dans sa Psychopathologie de la vie quotidienne, Freud a opposé ignorance consciente et connaissance inconsciente. C'est parce que le superstitieux ne sait rien de la motivation de ses propres actions accidentelles et parce que cette motivation cherche à s'imposer à sa reconnaissance qu'il est obligé de la déplacer en la situant dans un monde extérieur (1963, 298-9). La superstition n'est donc pas absurde, mais renferme un élément de vérité. Le superstitieux qui renonce à un projet, parce qu'en sortant de chez lui pour aller le réaliser il trébuche sur le seuil, se montre meilleur psychologue que l'incrédule. Car ce présage, qui est un acte manqué, témoigne de son désir inconscient que ce projet n'aboutisse pas.

Au tableau de la religion populaire, un terme supplémentaire, la magie, a été étudié très tôt comme un concept fondamental d'anthropologie sociale et religieuse. Marcel Mauss (1950, 3-141), en particulier, a noté l'extrême importance, dans les rites magiques, des conditions de temps, de lieu, de matières et d'instruments. Ces conditions sont toujours nombreuses et impératives. On ne peut rien changer à un rituel sans qu'il perde toute son efficacité. De ce formalisme découlent deux conséquences. D'une part les rituels magiques ont un caractère contraignant, ils s'imposent de manière impérative, et d'autre part, derrière ce formalisme contraignant, le contenu est beaucoup plus 
mobile, plus mouvant, si bien qu'on trouve à la fois un grand nombre de pratiques magiques destinées à un même but et des pratiques semblables pour des buts différents.

Dans cette perspective, Nicole Belmont (1979) a considéré les pratiques magiques et superstitieuses de la religion populaire comme possédant à la fois la diversité du rituel obsessionnel et la stéréotypie du rituel religieux. Elles ont un caractère moins privé que le cérémonial obsessionnel, mais moins public que le rituel religieux, et cela non seulement parce que ces pratiques étaient condamnées par l'Église. Par leur nature, si elles sont le plus souvent de pratique individuelle, elles sont d'origine sociale et communautaire puisqu' elles sont transmises par la tradition. L'obligation d'accomplir les actes compulsionnels serait donc équivalente à la contrainte exercée par les rituels de la magie et les pratiques superstitieuses de la religion populaire. Cependant ce caractère contraignant des pratiques magiques, qui se retrouve être identique à la fois dans les croyances et les pratiques superstitieuses, est ressenti non pas comme une force “externe," comme l'a cru d'abord Nicole Belmont, mais plutôt intérieure, à l'instar du cérémonial des obsessionnels qui s'impose à eux avec force comme une contrainte interne. Ce mécanisme de projection d'un savoir inconscient dans le monde extérieur s'applique aussi bien aux superstitions collectives, à ceci près qu'on a affaire ici à un ensemble de traditions, dans lequel l'individu n'a qu'à puiser, au lieu de s'adresser aux événements et au hasard. Le caractère contraignant des croyances et des pratiques magiques et superstitieuses de la religion populaire s'explique alors par ce même mécanisme de projection et de déplacement. Leur force de contrainte vient aussi bien de la tradition qui impose une norme que de l'inconscient. Ce qui émane de l'inconscient mais que la conscience censure, est projeté dans le monde extérieur, soit sous la forme d'obsession individuelle, soit comme un ensemble de croyances et de pratiques codifiées par la tradition.

\section{Les Actes de Croyance}

La croyance demeure toujours un mécanisme des plus importants dans tout phénomène religieux. Les philosophes, de David Hume à Bertrand Russell, tenaient même la croyance pour la plus énigmatique des opérations de l'esprit. Les anthropologues aussi, quoiqu'ils aient mis un certain temps à partager les interrogations philosophiques, ils ont fini par admettre le caractère hautement problématique de l'attribution d'une croyance à autrui. Ce n'est pas par hasard qu'au terme de superstition ressenti plus tard comme péjoratif, on a souvent substitué celui de croyance populaire. Cette expression, qui en anthropologie sociale a relayé le terme de survivance aussi, semble meilleure dans la mesure où elle débarrasse la notion de tout caractère péjoratif ainsi que de toute référence à un a priori idéologique qui renvoie le sens dans un passé révolu. Néanmoins, la représentation des croyances primitives ou populaires sous les traits d'une adhésion sans recul a commandé les modes d'interprétation qui ont successivement prévalu en anthropologie. On a vu tour à tour dans les croyances le produit obligé d'un défaut de rationalité, la manifestation d'un type spécifique d'activité mentale ou, plus récemment, dans les conceptions relativistes, intellectualistes et symbolistes, des essais rationnellement menés, mais inachevés, d'explication de l'ordre cosmique et du monde social. Or, après les réflexions critiques de Needham (1972), le concept de croyance ne saurait s'appliquer à une expérience ou à une attitude 
universelle, car la réalité des croyances n'est pas directement accessible à l'observation (Evans-Pritchard 1965). Il n'existe qu'un agrégat de significations contradictoires dont chacune, prise isolément, peut sans doute se déchiffrer dans les conceptualisations d'autres cultures mais dont il n'y a pas de raison de penser qu'aucune autre culture ne les combine différemment d'une façon aussi arbitrairement nécessaire.

Contrairement aux termes désignant la religion, il semble que dans le vocabulaire indo-européen existait un terme commun pour désigner la croyance, qui témoigne d'un sens très ancien et sans doute fondamental. La linguistique indo-européenne, qui nous vient encore une fois en aide, a dégagé le sens étymologique de la racine indo-européenne *kred, qui a donné le verbe credo en latin et dont le substantif attesté dans la littérature védique, šraddhâ, serait l'équivalent d'une “dévotion" des hommes à un dieu, par exemple pour une joute, au cours d'une lutte, d'une rivalité. La situation est telle d'un conflit entre les dieux, où les hommes interviennent en appuyant l'une ou l'autre cause. Dans cet engagement, les hommes donnent une partie d'eux-mêmes qui renforce celui des dieux qu'ils ont choisi d'appuyer. Cette "dévotion" permet la victoire du dieu qui est le champion et, en retour, elle confere au fidèle des avantages essentiels: victoire dans les luttes humaines, guérison des malades, abondance des récoltes, fécondité des femmes, etc. (Benveniste 1969,1: 176-9).

Le correspondant latin du substantif védique šraddhâ n'est pourtant pas celui que la concordance morphologique des verbes fait attendre. Antoine Meillet (1921) a montré que c'est le mot fides qui sert de substantif verbal à credo, c'est-à-dire que ce mot a dû être substitué à un plus ancien dérivé de *kred, dont il semble avoir subi l'influence. Cet autre terme intéresse plus particulièrement mon enquête. Il souligne avec plus de force le caractère de confiance, de crédit, de créance, par lequel nous adjoignons notre acceptation, notre approbation, à ce qui n'est qu'une impulsion à croire. Il est en même temps le lieu où la récupération chrétienne et le décentrement théologique ont été les plus spectaculaires.

La foi est un acte volontaire qui dépend de nous, un accueil, une acceptation que nous pouvons même refuser ou suspendre. Le sens premier du mot foi est historiquement la bonne foi, au sens moral, comme vertu de loyauté. Les mots latins fides (foi) et foedus (pacte, accord, alliance) proviennent d'une même racine indo-européenne, *beidh-, qui suggère d'une manière générale l'idée de confiance mise dans quelqu'un ou dans quelque chose. La foi est effectivement un engagement durable de la confiance, suivant des formes variables telles que parole donnée, promesse, profession de foi, serment, contrat, traité, alliance, conventions diverses.

Les notions véhiculés par l'ensemble de ces termes, telles qu'elles apparaissent à travers l'analyse comparative des significations lexicales, permettent de faire l'hypothèse, dans la perspective de la philosophie analytique et de la linguistique des actes du langage, que la croyance est inscrite dans le discours et qu'elle pourrait également s'interpréter de la même façon que tout autre acte de discours. Elle serait alors un ensemble constitué par différents actes analysables à plusieurs niveaux. À la base se situe un acte propositionnel, l'acte même de croire, qui est un acte "locutoire," c'est-à-dire utilisant des énoncés, expressions, pratiques et comportements ayant sens et référence. Ensuite on pourra distinguer un acte "illocutoire," qui est ce que l'on fait en croyant, autrement dit dans l'acte de croire on accomplit un autre acte constatif, assertif ou déclaratif, sous forme de croyance chargée d'une force illocutionnaire. Finalement, 
j'aimerais aussi parler de l'acte "perlocutoire" de croyance, qui est ce que l'on produit par le fait de croire.

Selon l'usage commun, au singulier et plus volontiers au pluriel, la croyance est un terme constatif par lequel on entend l'objet même de la persuasion commune ou de la conviction intime. La croyance, c'est ce que l'on croit et, pour autant que croire c'est être persuadé qu'une chose est vraie, réelle, on désignera communément par croyance les diverses conceptions de la réalité qui sont ainsi professées. Or, comme les croyances ont rapport à la vie des hommes, on entend aussi par croyances les règles spontanément reconnues pour la vie sociale ou individuelle. On trouve donc parmi les croyances des représentations théoriques et des représentations pratiques. Faisant la jonction entre ces deux groupes, on trouve des croyances qui présentent un caractère à la fois descriptif et prescriptif, par exemple les croyances concernant la destinée individuelle, le sort des défunts, la nature de la divinité et de ses rapports aux humains. En ce sens, la croyance se confond avec les conceptions religieuses, dans la mesure où celles-ci sont à la base des croyances portant sur l'existence de quelque réalité et des croyances portant sur le caractère de légitimité et d'obligation des règles de vie.

En tout cas, la croyance implique fortement l'engagement personnel du croyant, qu'il soit simple locuteur ou créditeur, dans la prétention à la vérité de ses énoncés, exprimés sous forme d'expressions verbales, de pratiques religieuses ou de comportements rituels. Or, cet engagement apparâit comme une qualité de l'énonciation même de la croyance. Le substantif védique šraddhâ suppose déjà une relation personnelle directe entre celui qui place quelque chose qui lui appartient et une personne divine ou humaine, ou une chose considérée comme puissante et susceptible d'aider. La croyance, qui tire sa signification dans les mêmes origines, est donc fondamentalement l'action même de croire, le crédit, la confiance accordée à quelqu'un, à quelque divinité, à quelque opinion. C'est alors le pôle subjectif de la persuasion ou de la conviction qui est ainsi souligné. Le mot, plus volontiers employé au singulier, dit alors l'engagement de l'homme dans la persuasion qu'il a qu'une chose est vraie ou réelle, que quelqu'un lui est fidèle, que la divinité est de son côté.

À ce titre, si l'acte illocutoire est une énonciation performative, ce que l'on fait en parlant, ou "quand dire, c'est faire," selon l'heureuse traduction de la formule de Austin (1962), une énonciation constative, du fait même qu'elle implique l'engagement du locuteur précisément sous forme de croyance ou d'assentiment se charge également d'une force illocutionnaire, soulignée par Searle (1970) et Vanderveken (1988). La croyance se charge alors de cette force illocutionnaire des actes de discours, au point que je pourrais extrapoler et admettre également que "quand on croit, on fait exister." L'acte de foi plus précisément est un acte illocutoire qui partage avec l'acte de discours cette caractéristique fondamentale qu'un énonciateur ne peut pas les accomplir sans essayer de faire savoir au destinataire qu'il les accomplit. La différence entre illocution et perlocution repose sur la présence, dans l'illocution, de la reconnaissance par l'interlocuteur de l'intention du locuteur de produire tel acte illocutoire. De la même façon, la croyance implique la reconnaissance de l'intention du croyant, locuteur ou créditeur, de produire tel acte de confiance. Ce caractère auto-implicatif n'est pas sans évoquer l'instance de discours de Benveniste (1966) et son caractère autoréférentiel. Dans une analyse qui joint "intention et signification" d'une manière très proche de la phénoménologie husserlienne, on ne saurait interroger quelqu'un ou lui donner un 
ordre sans chercher en même temps à ce qu'il se sache l'objet d'une interrogation ou d'un ordre. De la même façon, on ne saurait "faire confiance" ni "avoir confiance" sans chercher à établir avec autrui une relation réciproque de loyauté.

Du fait que la confiance se donne et se reçoit, la valeur perlocutionnaire de l'acte de foi oscille entre le sens actif de faire confiance (avoir foi) et le sens passif d'inspirer confiance (faire foi, être digne de foi, jouir d'un crédit, être fiable). Elle détermine entre les personnes une relation de reconnaissance réciproque qui oriente la confiance soit dans une direction active (la confiance que je fais ou que j'accorde), soit dans une direction passive (la confiance que j'obtiens ou que je mérite). Sous ces deux directions opposées, l'idée essentielle est que la foi reproduit les termes complémentaires et asymétriques d'une relation entre les personnes diversement concernées par les enjeux de la foi, entre celui qui donne sa parole (ou inspire confiance) et celui qui la reçoit (ou fait confiance). On notera que le sens actif de la confiance est du côté de celui qui reçoit. La parole donnée engage une relation entre la loyauté de l'un et la confiance de l'autre. En donnant sa foi, on se donne soi-même à reconnaitre. À Rome comme dans la Bible hébraïque, ce sont les normes de fiabilité, de loyauté, de fidélité qui apparaissent au premier plan. Dans tous les cas, ce sont les formes sociales de la reconnaissance qui permettent d'analyser le genre de relation confiante, perlocutoire, exprimé par la notion de foi. À l'âge classique la foi pose la base personnelle d'un lien social et sert à exprimer toutes les formes de loyalisme: la foi entre les hommes comme la foi envers les divinités. Elle est comme un pacte que l'on fait avec soi-même, avec autrui et avec Dieu, elle implique la loyauté dans les conventions et la fidélité aux engagements pris.

Le caractère essentiellement "ouvert" de ces actes, leur rapport nécessaire à leur propre communication rendent difficile de ne pas les considérer comme partie intégrante du sens des énoncés, expressions verbales, pratiques religieuses ou comportements rituels, au moyen desquels on les accomplit. Il se trouve ainsi que, de la même façon que l'accomplissement de tout acte illocutoire, l'accomplissement d'un acte de confiance contient nécessairement une qualification de l'énonciation. Donner un ordre, c'est d'une part prétendre que le destinataire est obligé d'effectuer une certaine action, et, d'autre part, prétendre qu'il y est obligé à la suite de l'énonciation véhiculant cet ordre. En ordonnant à quelqu'un de faire quelque chose, l'énonciateur attribue à sa propre parole le pouvoir de créer à son destinataire une obligation qu'il n'avait pas auparavant. Il en est de même pour la question. Poser à quelqu'un une question, c'est caractériser l'événement même constitué par la production des mots comme obligeant le destinataire à un certain type de comportement (en l'occurrence, répondre à la question posée). De la même façon, l'hypothèse selon laquelle il appartient au sens de l'énoncé de la croyance d'être un acte de foi implique que l'on tienne pour une partie intégrante de ce sens une qualification de l'événement constitué par l'apparition et l'énonciation de la croyance. Cet événement apparaît comme un acte perlocutoire, autrement dit comme créateur d'obligations, produisant une transformation juridique de la situation des partenaires de la croyance, en l'occurrence le rapport entre les hommes et les dieux.

Si selon Evans-Pritchard les croyances ne s'observent pas directement, "croire" c'est engager sa confiance dans un acte de foi manifesté envers un dieu, mais à charge de revanche, c'est-à-dire pour obtenir spécifiquement son aide. Quand on fait crédit à quelqu'un et qu'on lui accorde la confiance, c'est toujours en croyant ou admettant que 
certaines conditions seront réalisées. La foi oriente la confiance, mais elle l'oriente sur la base de croyances fermes, c'est-à-dire sous-tendues de forces illocutionnaires. Dans l'ensemble des rapports cultuels des hommes et des dieux il y a un contrat initial où on peut toujours opérer une distinction entre les offrandes que les hommes adressent aux dieux et les avis que les dieux envoient aux hommes. Ce sont deux mouvements en sens inverse, des hommes vers les dieux et des dieux vers les hommes, qui sont présents ensemble et qui définissent deux champs complémentaires de l'échange. Comme Dumézil (1980, 47-59) l'a explicitement montré dans la tradition romaine, la foi a un double domaine, l'un presque mystique, l'autre pleinement juridique. C'est parce que les actes de cultes, et principalement le sacrifice, sont à Rome des actes de commerce, l'exécution de contrats d'échange entre l'homme et la divinité. Les sacra sont nécessairement prolongés par les auguria ou signa (Dumézil 1987, 131-41). Leur automatisme est moins magique que juridique. Ils sont contraignants comme un pacte, du moins comme ce type de pacte implicite que Marcel Mauss a étudié dans son Essai sur le don, forme archaïque de l'échange (1950, 145-279).

\section{INTÉRIORISATION ET EXTÉRIORISATION}

L'inscription de la croyance dans le discours et l'échange fait que l'acte de foi comporte toujours une certitude de rémunération. Il s'agit d'une sorte de "gage," d'enjeu, parfois quelque chose de matériel mais qui engage aussi le sentiment personnel. Cette notion est investie d'une attitude mentale, interior state, pour reprendre l'expression d'Evans-Pritchard (1965), d'une force intérieure, la confiance, qui appartient à soi-même et qu'on déplace vers autrui, vers quelque chose d'extérieur, qui est souvent un être ou quelque chose de supérieur et de puissant. La foi et la croyance se situeraient donc paradoxalement plutôt du côté de la superstition et de la religion populaire que de la religion officielle. Elles sont un mouvement de déplacement vers l'extérieur, vers autrui ou vers une divinité, que ce soient les dieux de l'Antiquité ou le Dieu chrétien, l'un des Saints ou la Vierge, à qui l'on remet quelque chose, une offrande en argent, un cierge ou simplement des paroles, c'est-à-dire une prière, avec la certitude de retrouver en retour une restitution sous forme d'une faveur. C'est ainsi qu'on voit que la "superstition" n'est pas seulement une pure projection vers l'extérieur de tendances inconscientes. Cette relation est un lien de fidélité qui s'accompagne nécessairement d'un acte de confiance et d'une certitude de retour, qui peut souvent être ou devenir une espérance divine.

Il ne faut pas confondre l'expression habere fidem, qui signifie "avoir foi, avoir confiance" en quelqu'un ou en Dieu, avec celle que les habitudes actuelles de penser invitent à formuler en profitant des facilités que donne l'article en français: "avoir la foi." Cette dernière formule a un sens attributif plutôt que relationnel: on attribue à quelqu'un une conviction religieuse. On n'est que trop porté aujourd'hui à imaginer la foi et la foi religieuse plus particulièrement comme quelque chose que l'on a ou que l'on n'a pas. Les valeurs relationnelles sont plus fondamentales, même lorsqu'on privilégie le sens actif en direction d'autrui ou de la divinité. Originellement la foi, la bonne foi, était la rectitude qui conditionne l'existence et la validité morale d'un témoignage, quel qu'il soit. Alors que le christianisme a transformé l'idée essentielle de la foi afin d'en faire une croyance dont on témoigne la présence à l'intérieur de 
soi-même. C'est dès les premiers auteurs que le christianisme s'est efforcé d'imposer cette idée. Saint Augustin (Cité de Dieu 4: 19), par exemple, n'a cessé d'affirmer de façon énergique que l'autel de la foi est dans le cœur et quiconque est assez éclairé pour la posséder, que son meilleur et principal ouvrage est de faire croire au vrai Dieu ... ${ }^{4}$

Effectivement, l'idée religieuse de la foi et de la croyance est introduite secondairement par le christianisme et reprise ensuite par l'islam. Avec le christianisme, pour reprendre Hegel dans ses Leçons sur la philosophie de la religion, nous sommes en présence d'un objet double, l'un immédiat, l'autre intérieur, qui est ce que l'on veut signifier; celui-ci doit être distingué du premier qui est extérieur. À la faveur de cette double constitution, la représentation devient le siège de deux mouvements contraires: un mouvement d'extériorisation et un mouvement d'intériorisation. L'opposition entre religion officiellement instituée et religion populaire, spontanément pratiquée, dans sa signification profonde, serait alors une opposition entre intériorisation et objectivation. $\mathrm{La}$ religion populaire est un mouvement d'aliénation, d'objectivation et d'extériorisation, qui procède d'un déplacement, d'une projection à l'extérieur (vorstellen) du savoir interne et subjectif, dans la mesure où celui-ci est extériorisé et objectivé dans la représentation (Vorstellung), dans le corps de croyances et de pratiques érigées en traditions. L'ensemble de ces pratiques et croyances, dont l'intention et les modalités de leur nécessaire instauration nous sont données par les mythes de la création et des origines, devient ainsi objet de la conscience collective, un objet qui se pose indépendamment du corps social et qui se dote d'une rationalité propre. C'est le moment de l'aliénation où l'homme donne forme à l'essence divine, radicalement autre et radicalement lointaine. Cette aliénation qui est en même objectivation et extériorisation sera ensuite nécessairement suivie par l'exigence ou la promesse de se retrouver "réuni," "recueilli," "retranché" à l'intérieur de la ferveur (Andacht), qui s'exprime dans le culte établi et qui imprègne la mise en œuvre de l'institution de la religion. La religion instituée devient ainsi un mouvement d'intériorisation, de retour sur soi-même, une introjection imposée par les obligations externes et objectives, en ce sens que "l'homme écarte l'opposition et s'élève jusqu'à Dieu et à la conscience de son unité propre avec cette essence." Au bout du compte, l'objet extérieur se dépasse dans l'objet intérieur et s'abolit comme objet.

Les deux démarches procèdent du religieux. Elles sont à la fois complémentaires et opposées. Aucune religion ne peut être pure intériorisation ni extériorisation illimitée, elle ne peut être pure pratique projetée de l'intérieur ni imposée de l'extérieur non plus. Sinon, la religion serait non seulement cette extériorisation de l'absolu dans le sensible et dans l'histoire, mais aussi le lieu où meurt la représentation. Il existe sans doute un point de non-dépassement dans le mouvement d'intériorisation, par exemple, celui où la religion devient purement individuelle. Alors que ce que l'Église appelait "pratiques superstitieuses," comme l'a vu aussi Nicole Belmont (1979), était un facteur d'intégration sociale beaucoup plus actif que le mouvement contraire d'intériorisation qu'elle favorisait. En fait, le mouvement d'intériorisation dissout la religion plus que le mouvement de projection et d'objectivation qui lui est opposé et qui ne peut être que collectif.

Les termes objectivation et intériorisation ne sont donc pas interchangeables. Au contraire, ils renvoient à deux systèmes de représentation opposés, le premier situant le sens dans un principe cosmique, le second dans une représentation ontologique. Les 
deux systèmes ordonnent les mêmes éléments, mais l'ordonnancement est différent. Dans le premier système, il y a prévalence de l'ordre du monde sur les dieux et des dieux sur les hommes. Nous avons à faire aux religions du cosmos, à un polythéisme fonctionnel, à temporalité cyclique. Dans le second système, c'est un dieu unique, le Dieu du monothéisme, qui crée l'ordre du monde, auquel est asservie l'humanité. Nous sommes dans les religions du Livre. Il n'y a d'ordre universel et nécessaire que pour autant que Dieu s'en porte garant envers l'homme, sur fond d'une contingence du monde qui n'est autre que l'expression de la puissance divine.

\section{IDÉologies de Contestation ET DE LiBÉRATION}

Curieusement, les interprétations sociologiques se partagent dans deux camps qui ont aussi distingué, indifféremment, deux natures différentes et opposées de la religion. C'est au seuil du grand problème posé par la philosophie hégélienne de la religion, le problème de l'objectivation, que Marx va insérer sa critique de la religion, qui tournera aussi autour des notions d'objectivation et d'aliénation. Ce que Hegel a appelé objectivation est-il révélation ou aliénation? Plus précisément, l'objectivation de l'absolu, signification essentielle de la croyance, est-elle ou n'est-elle pas la même chose que l'aliénation de l'homme? Ce qui donne sens à la croyance, est-ce la manifestation de l'être offerte à notre célébration, ou la perte de soi de l'homme dans un autre qui appelle révolte et reconquête? Dans le monothéisme, la confiance en Dieu a pour fondement l'autorité de la révélation. Or la foi universaliste n'est pas seulement confiance, mais aussi obéissance à l'autorité divine. Elle risque de se confondre alors avec une pression sociale irresponsable.

Le caractère sociologique de la pensée de Marx s'est marqué par l'infrastructure sociale qu'il a donnée à toute sociologie. La religion a cependant un régime spécial: elle est l'expression de la misère humaine, mais une expression faussée qui se donne l'illusion de l'au-delà pour ne plus sentir les douleurs d'ici-bas. De ce fait elle doit être détruite comme toute espérance illusoire de "lendemains qui chantent," c'est-à-dire comme toutes les utopies. Conformément à la parole de Marx, selon laquelle la religion est "l'opium du peuple," il apparaissait évident que, non seulement la religion instituée est une force de nature conservatrice, mais qu'elle fait essentiellement partie du système d'asservissement au service des intérêts de la classe dominante. Aussi, l'école fonctionnaliste en anthropologie considérait-elle que la religion était, parmi d'autres, une force de nature conservatrice, un facteur d'équilibre au service d'un ordre social s'efforçant de se perpétuer (Firth 1964). Cette thèse a été soutenue avec vigueur par les historiens, les sociologues et les anthropologues d'orientation marxiste.

Pourtant, Engels lui-même prétendait que le développement rapide du christianisme des premiers temps prouve également que la religion peut avoir un rôle différent dans une société qui pratique l'esclavage. Il s'est complu en effet à voir dans certains mouvements religieux des préfigurations du communisme. Il a attiré l'attention sur un grand nombre de révoltes sociales religieusement inspirées, dont celle de Thomas Münzer est le prototype. Dans un passage remarquable de sa Contribution à l'histoire du christianisme primitif, il déclarait même qu'il existe un parallélisme évident entre le développement du christianisme au $2^{\mathrm{e}}$ siècle et les progrès du socialisme révolutionnaire au cours du $19^{\mathrm{e}}$ siècle. Le fait que, moins de trois cents ans après sa première apparition, 
le christianisme était devenu religion d'État dans l'Empire romain, “doit nous assurer que la victoire du socialisme est absolument certaine" (Marx and Engels 1960, 313). Dans un autre passage du même ouvrage, il établit un parallélisme direct entre l'esprit de chapelle du socialisme et celui des groupements chrétiens du christianisme des origines (1960, 327). En un mot, le socialisme d'Engels n'est lui-même autre chose qu'un culte millénariste.

On connaît effectivement que dès le début du $2^{\mathrm{e}}$ siècle avant Jésus-Christ, dans la région de la Méditerranée orientale, un mélange confus d'idéologies, d'origine persique, égyptienne, grecque, juive, avait donné naissance à toute une série de cultes et sectes millénaristes étroitement proches les uns des autres. Il n'y avait pas, à l'origine, une seule et unique Église, mais un grand nombre de sectes chrétiennes. Les historiens et les sociologues des cultes millénaristes (Cohn, Desroche, Lanternari, Thrupp, Worsley) ont par ailleurs unanimement indiqué que, dans le cas où l'on dispose d'une documentation précise, on voit que les croyances millénaristes sont presque toujours basées sur des notions qui étaient très répandues parmi les adhérents des sectes judéo-chrétiennes du premier siècle avant notre ère. Les cultes du cargo en Mélanésie, les danses de l'Esprit des Indiens d'Amérique du Nord, les croyances des révoltés Taiping dans la Chine du $19^{\mathrm{e}}$ siècle, reprennent tous, selon leurs caractéristiques propres, des thèmes du Livre biblique de l'Apocalypse.

Sous l'effet apparent des profondes études historiques et comparatives des mouvements millénaristes, les auteurs de gauche ont reporté par la suite toute leur attention sur le credo religieux des élus inspirés de Dieu et des différentes sectes protestantes présentées, désormais, sous la forme de facteurs efficaces concourant à une libération révolutionnaire. D'un bout du monde à l'autre, protestations ou révoltes sociales apparaissent à la fois amorcées et masquées dans une revendication religieuse. Une telle revendication implique, par choc en retour, les postulats d'une politique d'émancipation sociale, économique et nationale.

L'importance historique du messianisme vient de la conjonction de croyances religieuses et d'aspirations économiques et sociales qu'il a fréquemment réalisées. Il a joué ainsi un rôle toujours contestataire et souvent révolutionnaire. On a décelé des éléments millénaristes dans la plupart des révolutions, notamment dans la révolution ang- laise du $17^{\mathrm{e}}$ siècle, et même dans celles qui étaient a-religieuses ou antireligieuses, comme la Révolution française et la Révolution soviétique. Cette corrélation est particulièrement claire lorsque la révolte sociale se conjugue avec la lutte pour l'indépendance nationale. Le règne peut prendre également un aspect principalement politique. L'établissement de dynasties, l'achèvement de régimes ou l'apparition des nationalités s'accompagnent fréquemment de spéculations et de dimensions messianiques. Ce phénomène rejoint et prépare l'attribution de droits divins à l'autorité politique.

Devant la dualité des interprétations sociologiques, faudrait-il pour autant opposer un Marx radicalement irréligieux et un Engels accordant à la religion un rôle de souffle vers le socialisme? Henri Desroche (1962), parmi d'autres, a bien perçu qu'il y a une contradiction qu'Engels aussi bien que Marx posent entre religion et socialisme et qu'il faut faire sauter pour passer de celle-là à celui-ci. S'il y a entre les mouvements religieux et les mouvements révolutionnaires une discontinuité typologique, 
il faudrait voir chez Engels une "continuité génétique," qu'on pourrait volontiers qualifier plutôt de "lien dialectique," entre les uns et les autres, le socialisme étant obtenu par dépassement (Aufhebung) de la religion.

\section{Hiérarchisation du Divin et Structures de Médiation}

Cette corrélation dialectique entre religion et révolution semble constituer l'essentiel même du phénomène religieux, à condition qu'on cherche à interpréter la signification profonde de sa structure de représentation et de médiation. La religion n'est autre chose qu'un système médiateur de représentation entre le terrien dépourvu de puissance et l'omnipotence de Dieu situé dans l'empyrée, une médiation qui fait en sorte que la divine puissance céleste puisse intervenir en faveur de l'homme privé de pouvoir sur la terre. Complémentaires au niveau d'une prise en compte globale du phénomène religieux, sacra et signa ne sont pas pour autant loin de s'exclure mutuellement, comme s'excluent l'acteur et celui qui est agi. La structure de médiation peut fort bien alors être de forme hiérarchique. Le médiateur est un être humain, le prêtre sacrificateur en l'occurrence, qui agit en tant que représentant d'une congrégation profane, se plaçant sur un plan supérieur vis-à-vis de celle-ci, mais se trouvant dans une position d'infériorité vis-à-vis de la divinité. La structure religieuse peut tout aussi bien être d'un autre type et se présentant comme un modèle négateur de toute hiérarchie. C'est à la puissance sacrée elle-même qu'appartient l'initiative. Elle se manifeste en répandant sur les fidèles les dons de sa grâce et le croyant est inspiré de façon directe. Le charisme, ou la grâce divine, le touche sans intermédiaire et ne dépend aucunement de la plus ou moins grande efficacité d'un rituel célébré par un prêtre médiateur.

Selon une hypothèse structurale, argumentée par Edmund Leach (1973), le modèle hiérarchique de médiation religieuse correspond à la dogmatique des croyances orthodoxes, alors que le modèle négateur de toute hiérarchie, en des circonstances réelles, est étroitement associé aux croyances millénaristes et au développement des hérésies. L'un est susceptible de soutenir un pouvoir établi et hiérarchisé, alors que l'autre convient à une minorité déshéritée ou opprimée, cherchant à justifier sa révolte contre l'autorité établie. Entre les deux types de théologie existent des différences essentielles. Néanmoins, chacune des deux conceptions sociologiques du rôle de la foi religieuse, d'orthodoxie ou de subversion, n'est nullement exclusive ou contradictoire par rapport à l'autre. Il ne s'agit pas là de deux types de comportements fixés d'une façon définitive, mais d'un processus d'évolution dialectique. Les héritiers des promoteurs de la révolution deviennent avec le temps les adeptes et fidèles défenseurs d'une autorité légitime. Cependant, les différences d'aspects et de tonalités dont s'accompagne le passage de la novation au conservatisme, suivi d'un retour à l'innovation, constituent un processus complexe qui appelle une analyse historique et anthropologique détaillée des valeurs culturelles et des ensembles sociaux.

Les hommes veulent un dieu qui soit le leur et cela entraîne soit l'apparition de ce dieu dans un personnage (messianisme), soit son annonce imminente par un messager (prophétisme), soit l'avènement d'un règne ou d'un royaume antécédent ou conséquent à une telle apparition (millénarisme). Le messianisme peut être dominé par un projet de réforme religieuse, ecclésiologique ou culturelle, mais ce projet s'accompagne d'une 
abstention socioreligieuse plus ou moins radicale à l'égard du monde existant, au moins vis-à-vis des cultes dominants. Le millenium, temps de paix et de bonheur, représente souvent une société sans classes. Dans le monde lourdement et rigidement hiérarchisé du Moyen Âge, par exemple, la conception d'un millenium anarchiste, égalitaire, réalisant le thème folklorique d'un "monde à l'envers" (Bakhtine 1964), avait un pouvoir révolutionnaire très fort. Plus encore, le millenium devait être préparé par les pauvres en qui les millénaristes voient les instruments de l'eschatologie. En ce qui concerne la classe ouvrière aussi, la corrélation entre la misère et la religion, perçue et dénoncée par les marxistes comme positive, a été démontrée au contraire comme étant négative par les enquêtes empiriques (Isambert 1953). La classe ouvrière en révolte rejette un catholicisme trop lié aux nantis. Une prise de conscience sociale de la condition ouvrière conduit bien, dans la ligne de Marx, à un rejet de la religion, mais il faudrait préciser qu'il ne s'agit pas là d'un rejet de la religion en tant que telle, mais plutôt du rejet de l'orthodoxie religieuse.

En tout cas, lorsque le sentiment religieux est au service de fins subversives, il y a toujours une conception polythéiste ou hiérarchisée du divin qui s'impose dans le système théologique. Les fidèles d'une secte extrémiste, qu'ils soient prêtres ou simples croyants, devront pouvoir communiquer directement avec la divinité. Les prophètes entrent en conflit avec les techniciens des sacra, dont ils dévalorisent les interventions rituelles. En situation de monopolisation de la relation au divin par la classe sacerdotale, c'est dans l'espace alternatif des signa que ceux qui n'ont pas la parole peuvent la prendre. Le “désenchantement du monde" commence avec eux. La Rédemption n’est pas alors un don fait à l'humanité entière sous condition d'une soumission individuelle aux règles d'un ordre établi, c'est un privilège que Dieu accorde à ses élus, les saints, qui sont avec lui dans un rapport de communication directe et subjective.

Suivant ce modèle, une hiérarchisation visible dans la conception du divin va de pair avec une politique égalitariste dans la société des hommes. Dans la forme chrétienne de cette conception doctrinale, le fils de Dieu, le Christ-Logos, serait projeté dans un rôle subordonné par rapport à celui du Père divin, mais il sera plus proche de l'humanité et plus directement accessible. En quelque sorte, le Fils et le Saint-Esprit seront extériorisés au rang de demi-dieux. Les chefs spirituels de l'humanité seront eux-mêmes considérés à peu près comme des demi-dieux, étant donné qu'ils sont directement inspirés par le Saint-Esprit. En fait, pour de nombreuses sectes extrémistes et égalitaires, le Christ historique a été une sorte de superprophète bien plutôt qu'un Dieu, alors que tous les fidèles de la communauté sont subjectivement considérés comme des saints en puissance.

Cette même dialectique se trouve dans le monde de l'Islam aussi, quoique le sentiment religieux soit spécifique par rapport à la façon dont il est normalement exprimé dans la tradition catholique ou orthodoxe du christianisme. Une conception chère aux doctrines théologiques et régimes autocratiques islamiques est que l'Islam en tant que système religieux a atteint "l'unification religieuse" (tawhid al-dïn) par excellence en se présentant comme l'aboutissement universel des monothéismes spécifiques qui l'ont précédé. Pourtant, si le fait messianique a connu dans le judaïsme et dans le christianisme son développement le plus important et le plus original, certains ont pu voir dans l'islam même une branche du messianisme judéo-chrétien ou, du moins, de sa dimension apocalyptique concernant l'établissement d'un royaume de Dieu sans 
Église. En Islam, la relation du croyant avec Dieu est si personnelle qu'elle n'a pas besoin d'institution pour la médiatiser. Le catholique ne peut pas vivre sa foi sans passer par les mains du prêtre et sans accomplir certains rites, alors qu'il n'existe pas de sacrements pour le musulman. Sa relation avec le clergé est de nature sociale et non spirituelle. L'Islam ne reconnaît en principe aucun ordre religieux, aucun clergé, aucune hiérarchie spirituelle. Dans ces conditions, la conversion à l'Islam a dû probablement se fonder, au-delà d'une perception égalitaire des relations humaines, sur une conception hiérarchisée de la divinité. Le Coran était la parole de Dieu confirmant les autres Livres révélées, comme le prophète Mahomet, agent de la révélation, était le dernier de la série des messagers de Dieu qui commence par Adam et inclut, entre autres, Abraham, Moïse et Jésus, considéré comme un prophète parmi d'autres.

Toutefois, après un certain temps, les membres de la minorité anarchiste persécutée vont sûrement acquérir, dans l'ensemble social, une certaine respectabilité politique. À ce stade les doctrines de déplacement et d'extériorisation, subjectives et millénaristes, vont s'estomper et passer à l'arrière-plan. En fin de compte et selon toute probabilité, les héritiers des prédicateurs de l'hérésie deviendront les porte-parole d'une orthodoxie institutionnalisée dont le régime politique recherche l'appui. Dans l'histoire du christianisme, par exemple, une régression des croyances millénaristes se produisait en tous lieux où se structurait une hiérarchie ecclésiastique, et vice versa, chaque fois qu'une église schismatique proclamait son indépendance, cette option s'accompagnait d'une résurgence du millénarisme. Au $5^{\mathrm{e}}$ siècle, quand le christianisme était reconnu comme la religion officielle de l'État, sa doctrine révolutionnaire devenait hors de propos. Le Saint Augustin de la Cité de Dieu (20: 3-29) passa les dernières années de sa vie à soutenir les efforts des autorités politiques pour éliminer les millénaristes dissidents de l'hérésie donatiste en Afrique du Nord. Le même Saint Augustin qui, dans sa jeunesse, avait déjà éprouvé un certain attrait pour les croyances millénaristes, déclarait désormais que le Millenium du Messie n'était rien d'autre que la représentation symbolique de l'ère de la chrétienté (Brown 1972).

L'instauration politique d'une hiérarchie dans la société humaine s'accompagne de l'introjection d'un pur monothéisme dans le système théologique. Dans l'orthodoxie catholique, par exemple, la Rédemption de l'humanité est un événement historique qui appartient au passé et qui ne saurait se reproduire. La fusion des natures divine et humaine est réalisée en sa plénitude dans la personne du Christ. Il ne saurait y avoir de projection hiérarchique à l'intérieur d'une essence divine. Il existe un Dieu unique et lointain dont la légitimité, existant dès l'origine, est révélée et transmise par la parole des apôtres et de leurs successeurs. Seul un prêtre consacré peut en permettre l'approche, au moyen de la médiation objective par les sacra, dont la répétition infinie, au lieu qu'elle en dissolve le modèle, constitue celui-ci en élément d'une technique et d'un savoir relatif à cette technique. Max Weber (1971) n'a-t-il pas superbement montré, à propos du Judaïme antique, dans quelles conditions et selon quelles modalités peut se constituer une classe sacerdotale, en charge d'un tel savoir?

\section{DYNAMIQUE DES RECOMPOSITIONS IDENTITAIRES}

Au terme de cette analyse, pour reprendre à mon compte ces hypothèses, j'aimerais remarquer que l'opposition entre orthodoxie et subversion, intériorisation et objectiva- 
tion, religion officielle et religion populaire, religion dominante et religion dominée, a été le plus souvent ressentie comme une contradiction difficile, sinon impossible à surmonter. C'est particulièrement le cas des sociétés européennes, où existaient des discontinuités culturelles. L'élite dominante et le pouvoir établi essayaient en vain d'imposer une plus grande intériorisation de la religion, en contrôlant étroitement les processus d'extériorisation, c'est-à-dire en imposant une liturgie et un culte orthodoxes à la multitude considérée comme inculte. Celle-ci avait besoin de croyances et de pratiques qui manifestent plus adéquatement ses tendances et ses besoins profonds, donc de mécanismes de projection propres à elle, inventés par elle pour exprimer de manière collective, sociale, un savoir inconscient, censuré par la culture savante et dominante, qui souvent correspondait aussi à une culture étrangère.

Les religions populaires, de même que les religions antiques, sont des religions d'équilibre, pour reprendre l'expression de Van der Leeuw. Les rites y assurent une sorte d'échange de prestations entre Dieu et l'homme, entre la nature et la culture. L'expérience intérieure et subjectivée qui pourrait correspondre à ces rites, ne joue pas un rôle essentiel. Ces religions ne revendiquent donc pas la totalité de la vie intérieure de leurs adeptes et elles sont largement tolérantes, dans la mesure où elles admettent à côté d'elles une multiplicité d'autres rites et d'autres cultes.

L'expérience intérieure atteint son intensité la plus haute dans les religions de la “conscience malheureuse," comme les a qualifiées Hegel, c'est-à-dire dans les religions universalistes, dans lesquelles il y a rupture entre l'homme et la nature, dans lesquelles l'équilibre des échanges entre l'humain et le divin est rompu. Notamment, la conversion religieuse revêt dans ces religions un aspect radical et totalitaire, en prenant la forme d'une foi absolue et exclusive en la parole et en la volonté salvatrice de Dieu. Dans ces religions de "rupture," l'initiative de Dieu fait irruption dans le monde et introduit une nouveauté radicale dans le cours de l'histoire. La pérennité de l'ordre du monde est garantie à l'homme par Dieu au prix de son obéissance. La Parole que Dieu adresse à l'homme, consignée dans un livre sacré, exige une adhésion et une obligation absolues, une rupture totale avec le passé, une consécration et une introjection de tout l'être. Tel est l'enjeu du lien et de l'alliance au fondement du judéo-christianisme, forme exemplaire de monothéisme.

Ainsi, si le mouvement d'intériorisation dissout la religion au point où elle devient purement individuelle, l'individuation absolue dans la conception du divin fait perdre de la même façon tout caractère social à la religion, poussant à l'extrême la hiérarchie du système de médiation. C'est là que la communication est rompue et que, par conséquent, la religion se dissout. Le pouvoir religieux s'investit alors du pouvoir politique suprême pour ne laisser que deux alternatives possibles: le recours au millénarisme dans une intention de renversement de l'ordre établi ou bien le recours exclusif à la religion populaire et, dans certains cas extrêmes, à la conversion religieuse. Ce sont les seules échappatoires spirituelles, en vue d'un déplacement à l'extérieur des dispositions refoulées, ce qui est à la fois une appropriation par rapprochement du pouvoir dominant.

La conversion reflète plus particulièrement l'irréductible ambiguité de la réalité humaine. D'une part elle témoigne de l'expression et de l'extériorisation de la liberté de l'être humain, capable de se transformer totalement en réinterprétant son passé et son avenir. D'autre part, elle révèle que cette transformation de la réalité humaine résulte 
d'une invasion de forces extérieures, qu'il s'agisse de la grâce divine ou d'une contrainte psychosociale. Ainsi, quand on cherche à réinterpréter la religion dans sa relation aux valeurs locales, les conversions et reconversions successives tiennent lieu de mécanismes psychiques de déplacement et de projection au même titre que les mouvements messianiques et millénaristes. Les conversions permettent de dépasser les oppositions courantes entre monothéisme et polythéisme (ou paganisme, religion populaire, conception hiérarchisée du divin) en plaçant le religieux à son point de rencontre avec le politique: là où les croyances, les mythologies, les représentations du monde constituent l'édifice idéologique à partir duquel la société peut être pensée comme une totalité harmonieuse. Leur travail syncrétique implique non pas le mélange de deux systèmes religieux étrangers l'un à l'autre, mais l'appropriation d'une composante nouvelle de la religion qui privilégie une vision holiste de la société, aux dépens de l'idéologie individualiste dont l'orthodoxie religieuse était le promoteur.

Voilà pourquoi, au-delà des conditions historiques (crise, déstructuration, pression économique et sociale) qui favorisent l'apparition des conversions religieuses et tout autre type d'évangélisation, d'autres facteurs jouent un rôle important. Il s'agit notamment de l'existence d'une hiérarchie confessionnelle, d'une part, et de mythologies et religions populaires, d'autre part, qui charpentent l'univers socioculturel autour de figures divines et fondatrices hiérarchisées. Car il est tout à fait probable que paganisme ancestral et religion instituée, polythéisme et monothéisme, ont de tout temps évolué de concert, entretenant des rapports de tolérance, sinon même de connivence, jusqu'aux moments de l'affermissement absolu des pouvoirs et des orthodoxies ouvraient la voie aux millénarismes, aux schismes et aux conversions. À ces dates charnières, le double mouvement opposé de la religiosité, perçu comme antagoniste, cristallisait toujours une opposition sociale et culturelle. Le syncrétisme des conversions n'introduit pas alors une rupture dans l'ordre des conceptions religieuses. Il s'inscrit dans la permanence d'un mouvement régulier de retour aux fondements à la fois du politique et du religieux. Les mouvements de conversion religieuse, comme ceux de réforme spirituelle ou d'affirmation nationale, sont des créations originales et cohérentes. Leur apparition se veut une réponse dans l'imaginaire à une situation culturelle gravement altérée par l'intrusion brutale et angoissante de la domination idéologique, politique et étrangère.

\section{BIBLIOGRAPHIE}

Augé, Marc. Génie du paganisme. Paris: Gallimard, 1982.

Austin, John L. How to Do Things with Words. Cambridge, MA: Harvard University Press, 1962. (Trad. fr. Quand dire, c'est faire. Paris: Seuil, 1970, 1991).

Bakhtine, Mikhail. L'Euvre de François Rabelais et la culture populaire au Moyen Âge et sous la Renaissance. Paris: Gallimard, 1964.

Banton, Michael, ed. Anthropological Approaches to the Study of Religion. London: Tavistock, 1966.

Bastide, Roger. Les religions africaines au Brésil: contribution à une sociologie des interpénétrations de civilisation. $2^{\mathrm{e}}$ éd. Paris: PUF, 1995.

Bastide, Roger. Les problèmes de la vie mystique. Paris: PUF Quadrige, 1996.

Bastide, Roger. Éléments de sociologie religieuse. Nouv. éd. Paris: Stock, 1997.

Belmont, Nicole. "Superstition et religion populaire dans les sociétés occidentales," dans $\mathrm{La}$ fonction symbolique. Essais d'anthropologie, éd. M. Izard and P. Smith. Paris: Gallimard, 53-70, 1979.

Belmont, Nicole. Paroles païnnes, mythe et folklore. Paris: Imago, 1986. 
Benveniste, Émile. Problèmes de linguistique générale. Paris: Gallimard, 1966.

Benveniste, Émile. Le vocabulaire des institutions indo-européennes. 2 vols. Paris: Minuit, 1969.

Brown, Peter. Religion and Society in the Age of Saint Augustine. London: Faber, New York: Harper-Row, 1972.

Cohn, Norman. The Pursuit of the Millennium. London: Sacker \& Warburg, 1957. (Trad. fr. Julliard 1964, Payot 1983).

Desroche, Henri. Marxisme et religion. Paris: PUF, 1962.

Desroche, Henri. Dieux d'hommes. Dictionnaire des messies, messianismes et millénarismes de l'ère chrétienne. Paris-La Haye: Mouton, 1969.

Dumézil, Georges. Idées romaines. $2^{\mathrm{e}}$ éd. Paris: Gallimard, 1980.

Dumézil, Georges. La Religion romaine archaïque. $2^{\mathrm{e}}$ éd. revue et corrigée. Paris: Payot, 1987.

Eisenstadt, Samuel N., ed. Max Weber on Charisma and Institution Building, Selected Papers. Chicago University Press, 1968.

Evans-Pritchard, Edward E. Theories of Primitive Religion. Oxford: Clarendon Press, 1965. (Trad. fr. La Religion des primitifs à travers la théorie des anthropologues. Paris: Payot 1971).

Firth, Raymond. Essays on Social Organization and Values, London: Athlone, 1964.

Freud, Sigmund. La Naissance de la psychanalyse. Paris: PUF, 1956.

Freud, Sigmund. Psychopathologie de la vie quotidienne. Paris: Payot, 1963.

Freud, Sigmund. "Actions compulsionnelles et exercices religieux," dans L'Avenir d'une illusion. Paris: PUF, 81-94, 1976 [1907].

Freyburger, Georges. Fides. Étude sémantique et religieuse depuis les origines jusqu'à l'époque augustinienne. Paris: Belles-Lettres, 1986.

Hegel, G. W. F. Leçons sur la philosophie de la religion. Paris: PUF, 1996.

Isambert, François-André. "Classes sociales et pratique religieuse paroissiale." Cahiers Internationaux de Sociologie 14 (1953): 141-53.

Lanternari, Vittorio. Movimenti religiosi di libertà e di salvezza dei popoli oppressi. Milano: Feltrinelli, 1960 (Trad. fr. Maspéro, 1962).

Leach, Edmund. "Melchisedech and the Emperor: icons of subversion and orthodoxy," Proceedings of the Royal Anthropological Institute for 1972, 5-14, 1973. (Trad. fr. L'Unité de l'Homme et autres essais. Paris: Gallimard, 1980: 223-61).

Lebrun, Frédéric. "Le Traité des superstitions de J.-B. Thiers: contribution à l'ethnographie de la France du $17^{\mathrm{e}}$ siècle." Annales de Bretagne et des Pays de l'Ouest 83, no. 3 (1976): 443-65.

Levtzion, N., ed. Conversion to Islam. New York \& London: Holmes \& Meier, 1979.

Marx, K. and F. Engels. Sur la religion. Paris: Éditions sociales, 1960.

Mauss, Marcel. Sociologie et Anthropologie. Paris: PUF, 1950.

Meillet, Antoine. Mémoires de la Société de Linguistique de Paris 22 (1921): 215-18.

Middleton, John, ed. Gods and Rituals: Readings in Religious Beliefs and Practices. New York: Natural History Press, 1967.

Needham, Rodney. Belief, Language and Experience. Oxford: Blackwell, 1972.

Pereira De Queiroz, M. I. Réforme et révolution dans les sociétés traditionnelles. Histoire et ethnologie des mouvements messianiques. Paris: Anthropos, 1968.

Redfield, Robert. The Primitive World and its Transformations. Ithaca: Cornell University Press, 1953.

Saintyves, Pierre. "Les Origines de la méthode comparative et la naissance du folklore: des superstitions aux survivances," Revue de l'histoire des religions no. 105, (1932).

Searle, John R. Speech Acts: An Essay in the Philosophy of Language. Cambridge University Press, 1970. (Trad. fr. Les Actes de langage. Paris: Hermann, 1972).

Searle, John R. and Daniel Vanderveken. Foundations of Illocutionary Logic. Cambridge University Press, 1985.

Thrupp, Sylvia L. Millennial Dream in Action. La Haye: Mouton, 1962.

Van der Leeuw, G. La religion dans son essence et ses manifestations. Phénoménologie de la religion. Paris: Payot, 1948. 
Vanderveken, Daniel. Les actes de discours: essai de philosophie du langage et de l'esprit sur la signification des énonciations. Bruxelles: Mardaga, 1988.

Van Gennep, Arnold. Manuel de Folklore français contemporain. Paris: Picard, 1943.

Vernant, Jean-Pierre. "Les sciences religieuses et la sociologie," Cents ans de sciences religieuses en France. Paris: Cerf. 1987.

Weber, Max. Le Judaïsme antique. Paris: Plon, 1971.

Weber, Max. L'éthique du protestantisme et l'esprit du capitalisme. Paris: Plon, 1981.

Weber, Max. Sociologie des Religions. Paris: Gallimard, 1996.

Worsley, P. M. The Trumpet shall Sound: A Study of Cargo Cult in Melanesia. London: MacGibbon and Kee, 1957. (Trad. fr. Payot 1977).

\section{Notes}

1. Cet article est la version développée d'une communication destinée à être présentée dans la session Anthropological Theory and Anthropological Concepts at the End of the Century, 14th International Congress of Anthropological and Ethnological Sciences, College of William and Mary, Williamsburg, VA, 26 July-1 August 1998, http://www.wm.edu/ICAES/program/ mondaypm.html. À l'occasion, il m'a été impossible d'assister au Congrès de l'IUAES et le sujet de cet article fut discuté lors de deux communications que j'ai prononcées au Séminaire de Logique Symbolique et Idéologie à l'École des Hautes Études en Sciences Sociales à Paris et au Séminaire des Européanistes à l'Université de Paris-X à Nanterre. Je tiens à remercier Marc Augé et Emmanuel Terray d'une part, Georges Augustins et Martine Segalen d'autre part, ainsi que tous les participants, pour l'accueil favorable, les suggestions utiles et les discussions stimulantes qu'ils ont bien voulu m'accorder.

2. La coextensivité de la religion et de l'organisation sociale est un truisme ancien en anthropologie sociale. Plus exactement, si Marcel Mauss (1950, 145-279) a montré, à travers sa notion de "phénomène social total," que les principaux phénomènes sociaux ont une dimension religieuse comme ils ont une dimension économique, politique, esthétique..., beaucoup d'anthropologues ont travaillé sur ce sujet dans la plupart des sociétés, au point que la notion de "fait social total" est devenue un paradigme de base en anthropologie.

3. Envers la religion comme envers n'importe quelle expression humaine, Freud adopte le principe psychologique selon lequel l'homme y manifeste une vérité, sous une forme déplacée. Dans sa psychomythologie et dans L'Avenir d'une illusion, il envisage la religion du point de vue de l'individu et il l'explique d'après le modèle des rêves comme une création des désirs. Dans ses autres études, il considère la religion comme un mouvement transindividuel de la culture, mais obsédé par ses convictions de culpabilité œdipienne comme condition de la civilisation, il la centre sur la constitution de la loi éthique sous l'enseigne du père. Au début, dans la Naissance de la psychanalyse, Freud assimile la religion aux mythes et aux légendes, qu'il explique par la "projection," prise au sens large du terme. Ce sont des représentations de désirs pulsionnels, vaguement perçues de manière endopsychique, transposées dans l'extérieur et mettant ainsi en scène une réalisation de désirs interdits à l'homme tels que l'inceste, par exemple, ou contredits par la réalité telle l'immortalité. Dans les études sur la religion qui s'inspirent directement de la psychanalyse freudienne, les symboles religieux et mythiques sont l'objet privilégié d'une psychanalyse appliquée qui prend son modèle dans l'interprétation des images oniriques. Cette psychanalyse concerne le phénomène religieux dans la mesure où elle ramène l'intention symbolisante aux représentations archaïques que le symbolisme religieux viendrait soit refouler et recouvrir, soit assumer dans un processus de métaphorisation et de sublimation.

4. Il est piquant de voir, avec Dumézil $(1980,58)$, le saint polémiste d'Hippone, qui vivait si intensément sa fides-croyance, la chercher, sans bien entendu la trouver, dans la fides-loyauté du panthéon païen et traiter celle-ci avec autant de liberté que n'importe quelle divinité romaine. 